Current Science International

Volume: 10 | Issue: 01| Jan. - March| 2021

EISSN:2706-7920 ISSN: 2077- 4435

DOI: $10.36632 / \mathrm{csi} / 2021.10 .1 .20$

Journal homepage: www.curresweb.com

Pages: 217-225

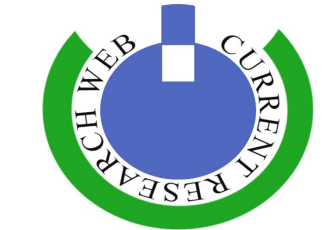

\title{
Early Retirement Phase and TMD
}

\author{
Amal H Moubarak B.D.S., M.Sc., Ph.D ${ }^{1,2}$ and Souna Saad El-Din B.D.S., M.Sc., Ph.D \\ ${ }_{1,2}$ Professor in Prosthodontics, Department of Oral and Maxillofacial Prosthodontics, King Abdulaziz \\ University- KSA.
}

Received: 25 January $2021 \quad$ Accepted: 20 March $2021 \quad$ Published: 25 March 2021

\begin{abstract}
Objectives: To evaluate the effect of retirement on Research Diagnostic Criteria for Temporomandibular Disorders (RDC/TMD) and to study the relation between retirement and TMDpsychological profile. Methods: Longitudinal study was done on 50 male retired volunteers. RDC/TMD tool which consists of axis I (physical assessment, 84-item questionnaire and clinical examination for TMJ and muscles) and axis II (psychosocial and pain-related disability assessments depending on symptom checklist 90 (SCL-90) was selected. Results: Axis I, the highest percentage of retirees suffering from muscle disorder (MD) before and after retirement. Axis II, Number of pain items (NPI) decreased after retirement when the number is between $0-3$ and it increased after retirement when it is 4-5. Graded Chronic Pain Scale (GCPS) moderate pain grade III increased after retirement from $18 \%$ to $30 \%$. Psychosomatic profile scores and percentages indicate moderate depression in contrast to severe depression before and after retirement. Conclusion: Percentage of retirees fallen into depression increased after retirement and suffering more from muscle disorders type of TMD. RDC-TMD is a valuable tool for diagnosing TMD with different subgrouping.
\end{abstract}

Keywords: RDC/TMD: depression, somatization, retirement, TMJ

\section{Introduction}

Retirement is meaningful life-event that changes everyday life style. The psychological process of retirement follows the same spontaneous emotional pattern and phases that are naturally accompanying other aspects of life.

Retirement has been defined as that process which begin with planning and preparing for, while workers are still at work (Wang and Shultz, 2010). Retirees should familiarize themselves with the landscape of their new circumstances and navigate their lives accordingly (Wang, 2007). The period of transition into retirement offers a unique opportunity for researchers to study adaption and coping over changes in psychological and mental health before, during, and following retirement (Zaniboni, et al., 2021\& Lee and Smith 2009).

In fact work may give meaning and a social context to peoples' lives and the absence of work may be difficult to deal with and may cause depressive response. Retirement by itself may not create depression (Lee and Smith 2009). However, the financial stress from losing job is a common stressful events for older adults (Lue et al., 2010). This stress can be also originated as a result of empowerment, emotional instability and heath condition (Abe, et al., 2012, Fernández-Niño, et al., 2018 \& Segel-Karpas, et al., 2018).

Epidemiologic studies that assess the process of human aging are very important, especially, when evaluating the oral health impact and its consequences in the elderly (Dahlström and Carlsson, 2010). Association between experiencing of stressful life events as retirement and muscle-related temporomandibular disorders has been reported. Psychological factors are known to play an important

Corresponding Author: Amal H Moubarak, Professor in Prosthodontics, Department of Oral and Maxillofacial Prosthodontics, King Abdulaziz University- KSA.

E-mail: amalscientific@yahoo.com 
role in the etiology and severity of temporomandibular disorders (Colaco et al., 2020 \& Yekkalam and Wänman, 2014).

Another factor as host susceptibility plays a role in TMD. Genetically influenced physical traits determine disease onset and its progression. In addition, environmental parameters such as ethnicity, culture and stress are essential variables in the patient's susceptibility and demand for treatment (de Kanter, et al., 2018, Gillborg, et al., 2017 \& Reiter, et al., 2006).

Depression and chronic widespread pain are risk factors for orofacial muscle pain. Studies suggest that stress-related disorders may contribute to the development of TMD chronicity and may therefore be viewed as perpetuating rather than initiating factors (Reiter, et al., 2015).

The Research Diagnostic Criteria for Temporomandibular Disorders (RDC/TMD) specifies a dual-axis diagnostic system for temporomandibular disorders (TMD) supported by a welloperationalized history and examination protocol. The Axis I clinical assessment protocol is designed to render TMD diagnoses, and the Axis II screening instruments assess psychological status and painrelated disability. Together, Axis I and Axis II assessments constitute a comprehensive evaluation consistent with the biopsychosocial health model. (Schiffman, et al., 2014).

The aim of this study was to evaluate the effect of early phase of retirement on TMD by RDC including psychosomatic profile.

\section{Material and Methods}

Fifty male retired volunteers (58-60 years) were participated and informed about the study and gave them informed consent. Criteria for exclusion from the study were the followings: (a) major physical illness (b) presence of polyarthritis or other rheumatic diseases; (c) acute dental pain. Data were collected twice, 6 months before and after retirement. At the beginning, all participants who reported at least two of the cardinal signs or symptoms of TMD: Jaw pain, limited mouth opening, muscle pain or TMJ noise were diagnosed as non- RDC/TMD patient.

For RDC/TMD measurements, the data collection was done by one investigator to assess TMD signs and symptoms as suggested by the literature (Schiffman, et al., 2014). Distribution of the Arabic version of (RDC/TMD) questionnaire (87 items) on study participants was achieved including the dual axis system. Then, a clinical examination of TMJ and the surrounding muscles was done according to the standardized published specifications (https://ubwp.buffalo.edu).

1. Scoring of the findings for subsequent grouping and subgrouping according to Axis I criteria into 3 categories as shown in table 1 .

2. Asses the level of the psychosomatic status of participants and the TMD related psychological disabilityaccording to Axis II criteria.

Subjects completed the RDC/TMD Axis II self-report measures, which include the Graded Chronic Pain Scale (GCPS) which depends on the Characteristic Pain Intensity (CPI) and Jaw Disability Checklist, and the Revised Symptom Checklist (SCL-90-R) (Derogatis, et al., 1973 \& Osiewicz, et al., 2018).

GCPS domain is composed of six items assessed (Q7:9 and 11:13) on a 10-point scale, and one item on the number of disability days (Q10) due to facial pain and scoring criteria are simple to use. It assesses pain intensity, interferences with usual activities, family and leisure activities, work-related activities. Then, assessment of the psychological status using mean depression and somatization scores from subscales of the Symptom Checklist-90 (SCL-90) (Derogatis, 1983).

The psychological status was assessed through depression (DEP) and non-specific physical symptom (somatization SOM) scores measured with subscales of SCL-90-R. The questionnaire included 32 questions with 5-point Likert response scale (ranged from 0 to 4), 12 items of somatization subscale and 20 items of depression subscale with total of 32 items. The domain was derived from items 20 (a, c, d, j, o, p, r, s, t, u, w and x) when pain items were excluded, the remaining domain consisted of items $20(\mathrm{c}, \mathrm{r}, \mathrm{s}, \mathrm{t}, \mathrm{u}, \mathrm{w}$ and $\mathrm{x})$ finally the domain for depression was derived from items 20 (b, e, f, g, h, I, k, l, m, n, q, v, y, z, aa, bb, cc, dd, ee, ff.) . The five nonspecific pain conditions examined were incorporated into the SCL-90, and patients were considered to have a pain item if they responded "moderately" to "extremely" when asked how much the pain item distressed them in the last month (Dworkin, and Leresche, 1992, Yap, et al., 2002 \& Manfredini, et al., 2011). 
Table 1: RDC/TMD groups and subgroups for axis I

\begin{tabular}{ll}
\hline Groups & Subgroups \\
\hline \multirow{2}{*}{ Group I: muscle disorders(MD) } & $\begin{array}{l}\text { Ia. Myofascial pain. } \\
\text { Ib. Myofascial pain with limited opening. }\end{array}$ \\
\hline \multirow{3}{*}{ Group II: disc displacements(DD) } & $\begin{array}{l}\text { IIa. Disc displacement with reduction. } \\
\text { IIb. Disc displacement without reduction with limited opening. } \\
\end{array}$ \\
$\begin{array}{ll}\text { IIc. Disc displacement without reduction with limited opening. } \\
\text { Group III: arthralgia, osteoarthritis, } \\
\text { osteoarthrosis(AAA) }\end{array}$ & $\begin{array}{l}\text { IIIa. Arthralgia. } \\
\text { IIIb. Osteoarthritis of the TMJ. }\end{array}$ \\
& IIIc. Osteoarthrosis of the TMJ.
\end{tabular}

Characteristic Pain Intensity (CPI) was evaluated through scoring items in the questionnaire about pain history. The patient's score ranged from 0 to 100 , with 100 being the most intense pain. Disability Points was calculated as the sum of points for disability days and points of disability score.

The GCPS was used to classify individuals according to Characteristic Pain Intensity and Disability Points on grades (I to IV). The detailed description of each grade is shown in table (2). The DEP and SOM mean scale score is calculated by summing up the score of the single items. This makes possible to rate patients as having normal, moderate or severe levels of impairment in the depression and non-specific physical symptoms scales. The limit of each is shown in table (2) (Manfredini, et al., 2006 \& Manfredini, et al., 2010).

Table 2: Grading scores for Axis II

\begin{tabular}{cl}
\hline Item & Description \\
\hline & Grade 0: no TMD pain in the previous 6 months \\
& Grade I Low disability: Low Intensity Characteristic Pain \\
& Intensity $<50$, andless than 3 Disability Points \\
& Grade II : High Intensity Characteristic Pain Intensity $>50$, \\
& andless than 3 Disability Points \\
& Grade III High Disability: Moderately Limiting 3-4 Disability \\
& Points, regardless of Characteristic Pain Intensity \\
& Grade IV: Severely Limiting 5 -6 Disability Points regardless of \\
& Characteristic Pain Intensity \\
& no $(\leq 0.535)$ \\
& Moderate $(0.535-1.105)$ \\
& Severe $(\geq 1.105)$ \\
\hline \multirow{3}{*}{ DEP } & no $(\leq 0.500)$ \\
& Moderate $(0.500-1.00)$ \\
& Severe $(\geq 1.00)$ \\
\hline \multirow{3}{*}{ SOM(with pain items included) } & no $(\leq 0.428)$ \\
& Moderate $(0.428-0.857)$ \\
& Severe $(\geq 0.857)$ \\
\hline
\end{tabular}

Collected data were statistically analyzed. Calculation of mean and standard deviation and comparison through Chi-squared test at the 5\% level of confidence were performed using SPSS foundation for statistical computing (Vienna, Austria. ISBN3-900051-07-0).

\section{Results}

The mean age of our participants was 59.1 years. Before retirement (BR), regarding Axis I $(84 \%)$ had no MD, subgroup Ia was found in (10\%) and subgroup Ib was found in (6\%).

Group II DD, $4-2 \%$ of the participants demonstrated subgroup IIa and IIbs respectively in the right side (RT) of the joint. $2 \%$ demonstrated subgroup IIa in the left side (LT) of the joint.

Group III AAA, no participants demonstrated any of the in the right side of the joint. Only $2 \%$ demonstrated subgroup IIIa in the left side of the joint. The total number of TMD before retirement was $26 \%$. 
After retirement (AR), regarding Axis I (80\%) had no MD, subgroup Ia was found in $(14 \%)$ and subgroup Ib was found in (6\%).

Group II DD, no change was found in the percentage in the right side (RT) of the joint. Raising into 4\% was found in subgroup IIa in the left side (LT) of the joint.

Group III AAA, no change was found in the percentage in both sides. The total number of TMD before retirement was $32 \%$ as shown in table (3) \& fig (1).

Table 3: The distribution of the participants according to axis I groups and subgroups.

\begin{tabular}{|c|c|c|c|c|c|c|c|}
\hline Group & I MD & & II DD & & III AAA & & Total \\
\hline \multirow{4}{*}{ BR } & Ia & $\mathrm{Ib}$ & RT & LT & RT & LT & \multirow{4}{*}{13} \\
\hline & \multirow{3}{*}{5} & \multirow{3}{*}{3} & IIa 2 & IIa 1 & IIIa 0 & IIIa 1 & \\
\hline & & & IIb 1 & IIb 0 & IIIb 0 & IIIb 0 & \\
\hline & & & IIc 0 & IIc 0 & IIIC 0 & IIIc 0 & \\
\hline \multirow{4}{*}{$\mathbf{A R}$} & Ia & $\mathrm{Ib}$ & RT & LT & RT & LT & \\
\hline & \multirow{3}{*}{7} & \multirow{3}{*}{3} & IIa 2 & IIa 2 & $\begin{array}{l}\text { IIIa } 0 \\
\end{array}$ & IIIa 1 & \multirow{3}{*}{16} \\
\hline & & & IIb 1 & IIb 0 & IIIb 0 & IIIb 0 & \\
\hline & & & IIc 0 & IIc 0 & IIIC 0 & IIIc 0 & \\
\hline
\end{tabular}

TMD Groups and subgroups distribution before and after retirement

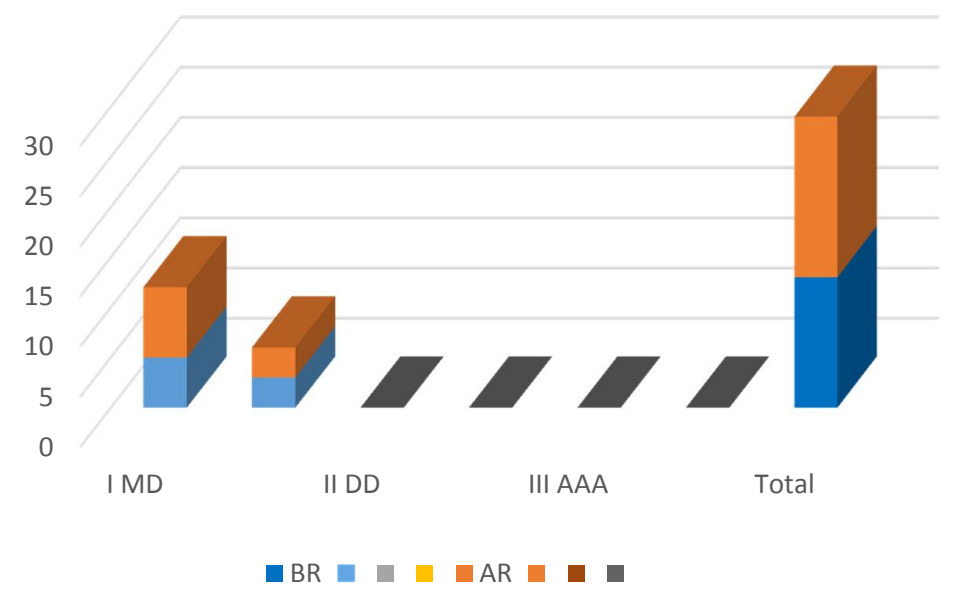

Fig. 1: TMD groups and subgroups distribution before and after retirement (MD: muscle disorder DD: disc displacement AAA: arthralgia, osteoarthritis, osteoarthrosis)

In Axis II, Number of pain items (NPI) before and after retirement decreased (-) after retirement when the number varied from 0 to 3 items and adversely it increases ( + ) after retirement when the number varied from 4 to 5 items as shown in table 4 .

Table 4: Number of pain items (NPI) before and after retirement

\begin{tabular}{ccccccc}
\hline NPI & $\mathbf{0}$ & $\mathbf{1}$ & $\mathbf{2}$ & $\mathbf{3}$ & $\mathbf{4}$ & $\mathbf{5}$ \\
\hline BR & 9 & 7 & 5 & 4 & 15 & 10 \\
AR & 6 & 6 & 4 & 3 & 17 & 14 \\
$(+)(-)$ & $(-)$ & $(-)$ & $(-)$ & $(-)$ & $(+)$ & $(+)$ \\
\hline
\end{tabular}

Regarding GCPS which denotes pain level, mild pain expressed by grades I\&II has been ranged from $24-23 \%$ respectively, while moderate pain grade III increased after retirement from $18 \%$ to $30 \%$. Also, severe pain grade IV increased after retirement to reach $4 \%$ in contrast to $0 \%$ before retirement. These data are presented in table 5 and figure 2 
Table 5: Chronic pain grade scale (GCPS) before and after retirement

\begin{tabular}{cccccc}
\hline GCPS & 0 & I & II & III & IV \\
\hline BR & 17 & 13 & 11 & 9 & 0 \\
AR & 10 & 11 & 12 & 15 & 2 \\
\% & 27 & 24 & 23 & 24 & 2 \\
\hline
\end{tabular}

Chronic pain grade scale (GCPS) before and after retirement

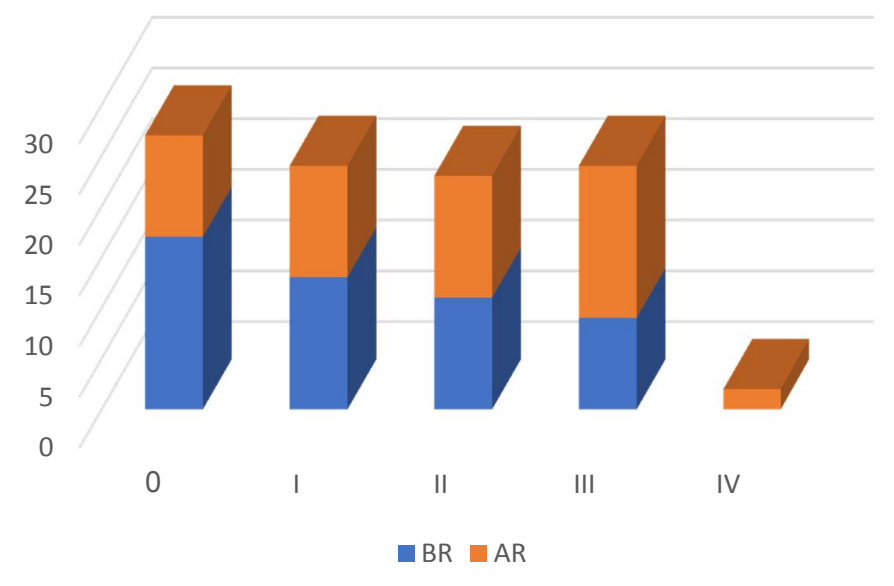

Fig. 2: Chronic pain grade scale (GCPS) before and after retirement

Axis II psychosomatic profile scores and percentages indicate moderate depression with mean value of (0.973) in contrast to severe depression with mean value (1.122) before and after retirement and increase the percent of retirees fallen into depression from $12 \%$ to $22 \%$ after retirement. In regard to depression, there is statistically significant difference with P-value of (0.047) between volunteers before and after they retired.

In contrast, somatization with or without pain showed increase in the mean values and percentages of both moderate and severe levels after retirement but within the range of moderate values. Regarding somatization, no statistically significant difference between volunteers before or after they retired. Psychosomatic items arepresented in table 6 and figure 3

Table 6: Psychosomatic profile before and after retirement

\begin{tabular}{ccccc}
\hline Psychosomatic profile & & BR & AR & BR\%-AR\% \\
\hline & No & 34 & 32 & $68 \%-64 \%$ \\
Depression (DEP) & Moderate & 10 & 7 & $20 \%-14 \%$ \\
& Severe & 6 & 11 & $12 \%-22 \%$ \\
& $\mathrm{M} \pm \mathrm{SD}$ & $0.973 \pm 0.10$ & $1.122 \pm 0.23$ & \\
\hline & No & 36 & 33 & $72 \%-66 \%$ \\
Somatization & Moderate & 9 & 11 & $18 \%-22 \%$ \\
(SOM-with pain) & Severe & 5 & 6 & $10 \%-12 \%$ \\
& $\mathrm{M} \pm \mathrm{SD}$ & $0.533 \pm 0.3$ & $0.811 \pm 0.4$ & \\
\hline & No & 36 & 32 & $72 \%-64 \%$ \\
Somatization & Moderate & 8 & 10 & $16 \%-20 \%$ \\
(SOM-without pain) & Severe & 6 & 8 & $12 \%-16 \%$ \\
& $\mathrm{M} \pm \mathrm{SD}$ & $0.567 \pm 0.4$ & $0.664 \pm 0.2$ & \\
\hline
\end{tabular}




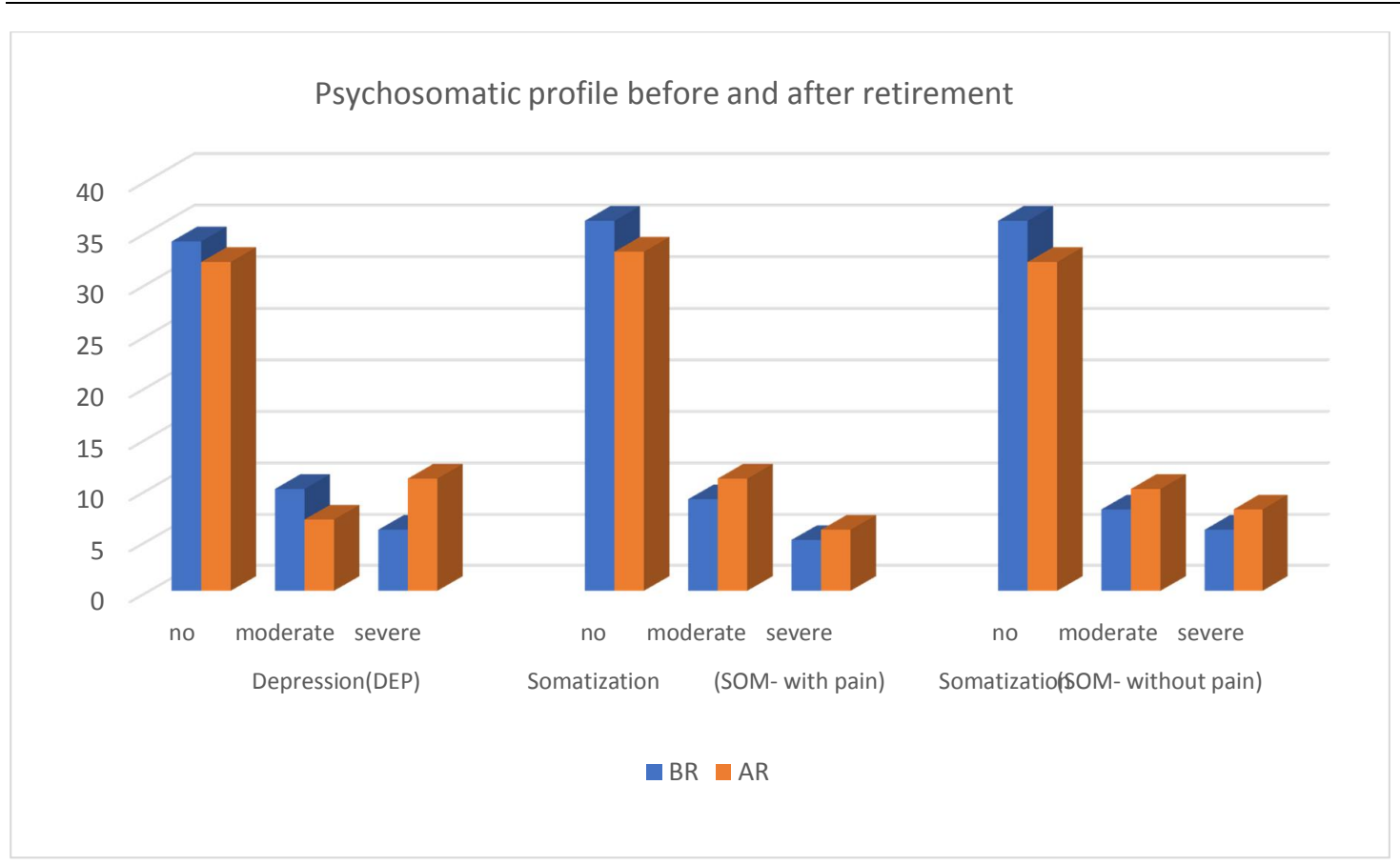

Fig. 3: Depression and somatization scores before and after retirement

\section{Discussion}

One of the primary aims of the implemented RDC/TMD classification system was to facilitate cross-population comparison between different investigations and to increase knowledge on TMD epidemiology and to circumvent confusion generated by the use of multiple terms to indicate the same disorder.( Schiffman, et al., 2014). The better standardized capability and reliability of RDC tool in comparing TMDs groups is appreciated in many studies (Manfredini, et al., 2011, Ohrbach, et al., 2008, Steenks, and De Wijer, 2009 \& Karatas, O., et al., 2013). To our knowledge, this is the first study to examine the relationship between RDC/TMD axis I and axis II findings in retirement period.

TMD symptoms have a prevalence peak between 30-35 years of age and another peak 50-55 years old (Guarda-Nardini, et al., 2012). This finding is in consistence with our result as volunteers' average age 59.1 years demonstrated high percentage up to $26-32 \%$ in comparison to middle age which has less percentage $18-30 \%$.

Our study denotes that most of our participants belong to group I muscle disorder before and after retirement. This is quite similar to work done by Osiewicz et al., (2018) who found the prevalence of group I is more than the other two groups. Group II disorders was found in $9 \%$ of patients, most of them were diagnosed with disc displacement with reduction. This high frequency agrees with other studies done by John and Reissmann (John, et al., 2007; Manfredini, et al. 2011). This was followed by, group III inflammatory-degenerative disorders subgroup in about $2 \%$, with arthralgia being the most frequent diagnosis. Up to our knowledge, only few studies reported the prevalence of each subgroup diagnosis, thus limiting the possibility to discuss the findings in the general population in depth (Manfredini, et al. 2011).

Regarding DD with reduction, the range was from $6-8 \%$, while the two subgroups of DD without reduction had a lower frequency range $0-2 \%$. The observation that disc displacement is common, has been suggested by many authors (Nguyen, et al., 2017 \& Winocur, et al., 2009) such findings, along with the well-described high variability of disc position in asymptomatic subjects as well (Anderson, et al., 2010), lend support to the hypothesis that disc displacement can sometimes be considered a non-pathologic or, at least, a non-treatment- requiring condition .

Nearly $63 \%$ of our participants complained of three or more pain items as proved in table 4 which may be attributed to the elevated levels of depression/somatization. Linking multiple pains to the somatic expression of psychiatric and psychosocial disturbance is supporting our results which is logically being more noticeable in elder age group (Yap et al., 2002; Reiter et al., 2018; Valesan et 
al., 2021).

Our results revealed nearly $24 \%$ had mild to moderate psychosocial dysfunction of grades I, II and III, and 2\% had severe grade IV on GCPS (Reiter, et al., 2006 \& Yap, et al., 2002). Studies done on Swedish, American and Jewish populations demonstrated average percentage between $13-20 \%$ of grade III CPS and also found that Arab people had higher values of dysfunctional activity which is in concurrence to our findings (Gillborg, et al., 2017 \& Reiter, et al., 2006) .

This can be explained by the less quality of health care and profound referral to ENT specialties rather than TMD ones. There is a similar matching results regarding diminished percentage of grade IV in many earlier studies (Song, et al., 2018 \& Pigozzi1, et al., 2021).

Our results regarding DEP scores are higher than those found in other studies. This diversity in percentage might be attributed to differences in ethnic, race, social, psychological, cultural, and political nature (Reiter, et al., 2006). On the other hand our results are similar to that reported for Arabian females in the study (DEP 60\%) by Reiter et al. (2006). TMD and depression were interrelated in many studies (Lue et al., 2010; Abe et al., 2012; Fernández-Niño et al., 2018; SegelKarpas, 2018). As stress has been reported as a provoking factor for TMD (Nassif et al., 2003).

Before retirement, a moderate level of depression and somatization was revealed as shown in tab.6. The major sources of stress might be related to their age-related problems like health status, sleep disorders, drugs taken which exacerbated by stress anxiety accompanying fear of loss of financial resources and change in their life styles. Depression scores have been increased significantly after retirement due to the added psychological stress expressed by remarkable increase in nonspecific physical symptoms scores which could possibly explains the condition (Celić et al., 2011) . Our findings call attention to the need for clinical research with RDC accompanying further progression into retirement and the importance of prevention and intervention of stress amongst retirees.

\section{Conclusion}

Positive relation between TMD and early retirement phase when comparing data by RDC/TMD. Higher scores of muscle disorder, depression and somatization are clear in participants after retirement

\section{References}

Abe, Y., N. Fujise, R. Fukunaga, Y. Nakagawa, and M. Ikeda, 2012. Comparisons of the prevalence of and risk factors for elderly depression between urban and rural populations in Japan. Int Psychogeriatr, 24:1235-1241

Anderson, G.C., et al., 2010. The Research Diagnostic Criteria for Temporomandibular Disorders VI: future directions. J. Orofac Pain, 24: 79-88.

Celić, R., V. Braut, and N. Petricević, 2011. Influence of depression and somatization on acute and chronic orofacial pain in patients with single or multiple TMD diagnoses. Coll. Antropol., 35: 709-713.

Colaco J, et al., 2020. Oral health-related quality of life and associated factor in the elderly: a population-based cross-sectional study. Ciên. \& Saúde Colet., 25: 3901-3912.

Dahlström, L., and G.E. Carlsson, 2010. Temporomandibular disorders and health-related quality of life. A systematic review. Acta. Odontol. Scand., 68: 80-85.

de Kanter, R.J., P.M. Battistuzzi, and G.J. Truin, 2018. Temporomandibular Disorders: "Occlusion" Matters!: Review Article. Pain Res. Manag., ID8746858, 1-13.

Derogatis L.R., 1983. SCL-90-R. Administration, scoring and procedures. Manual - II. Towson, MD: Clinical Psychometric Research.

Derogatis, L.R., R.S. Lipman, and L. Covi, 1973. SCL-90: an outpatient psychiatric rating scalepreliminary report. Psychopharmacol Bull., 9: 13-28.

Dworkin, S.F., and L. Leresche, 1992. Research diagnostic criteria for temporomandibular disorders: review, criteria, examinations and specifications, critique. J. Craniomandib Disord, 6: 301-355.

Fernández-Niño, A.J., J. Laura, L.J. Bonilla-Tinocob, B.S. Manrique-Espinozac, M. RomeroMartínezc, and A.L. Sosa-Ortizd, 2018. Work status, retirement, and depression in older adults: An analysis of six countries based on the Study on Global Ageing and Adult Health (SAGE): SSM-Population Health, 6:1-8. 
Gillborg, S., S. Åkerman, N. Lundegren, and E. Ekberg, 2017. Temporomandibular Disorder Pain and Related Factors in an Adult Population: A Cross-Sectional Study in Southern Sweden. J. Oral Facial Pain Headache, 31, 37-46.

Guarda-Nardini, L., F. Piccotti, G. Mogno, L. Favero, and D. Manfredini, 2012. Age-Related Differences in Temporomandibular Disorder Diagnoses. Cranio, 30:103-9.

https://ubwp.buffalo.edu/rdc-tmdinternational/tmd-assessmentdiagnosis/rdc-tmd/

John, M., D. Reißmann, O. Schierz and R. Wassell, 2007. Oral Health-Related Quality of Life in Patients with Temporomandibular Disorders. J. Orofac. Pain, 21:46-54.

Karatas, O., et al., 2013. Identifying potential predictors of pain-related disability in Turkish patients with chronic temporomandibular disorder pain. J. Headache Pain, 14: 1-9

Lee, J., and J.P. Smith, 2009. Work, Retirement, and Depression. J. Popul. Ageing, 1: 57-71.

Lue, B.H., L.J. Chen, and S.C. Wu, 2010. Health, financial stresses, and life satisfaction affecting late-life depression among older adults: A nationwide, longitudinal survey in Taiwan. Arch Gerontol Geriatr, 50: S34-S38.

Manfredini, D., E. Winocur, J. Ahlberg, L. Guarda-Nardini, and F. Lobbezoo, 2010. Psychosocial impairment in temporomandibular disorders patients. RDC/TMD axis II findings from a multicentre study; J. Dent., 38: 765-72.

Manfredini, D., G. Chiappe, and M. Bosco, 2006. Research diagnostic criteria for temporomandibular disorders (RDC/TMD) Axis I diagnosis in an Italian Patients: J Oral Rehabil, 33: 551-558.

Manfredini, D., L. Guarda-Nardini, E. Winocur, F. Piccotti, J. Ahlberg, and F. Lobbezoo, 2011. Research diagnostic criteria for temporomandibular disorders: a systematic review of axis I epidemiologic findings. Oral Surg Oral Med Oral Pathol Oral Radiol Endod., 112: 453-62.

Nassif, N.J., F. AL-Salleeh, and M. AL-Admawim, 2003. The prevalence and treatment needs of symptoms and signs of temporomandibular disorders among young adult males. J. Oral Rehabil, 30: 944-950.

Nguyen, M.S., et al., 2017. Symptoms and signs of temporomandibular disorders among elderly Vietnamese. Proc. Singapore Health, 26: 211-216.

Ohrbach, R., J. Sherman, C. Beneduce, K. Zittel-Palamara, and Y. Pak, 2008. Extraction of RDC/TMD subscales from the symptom check list-90: does context alter respondent behavior? J. Orofac Pain., 22: 331-339.

Osiewicz, M.A., F. Lobbezoo, B.W. Loster, J.E. Loster, and D. Manfredini, 2018. Frequency of temporomandibular disorders diagnoses based on RDC/TMD in a Polish patient population. Cranio, 36: 304-310.

Pigozzi1, L.B., et al., 2021. Quality of life in young and middle age adult temporomandibular disorders patients and asymptomatic subjects: a systematic review and meta-analysis Health Qual Life Outcomes, 19: 1-22.

Reiter, S., A.E. Perlman, C. Goldsmith, P. Friedman-Rubin, and E. Winocur, 2015. Comorbidity Between Depression and Anxiety in Patients with Temporomandibular Disorders According to the Research Diagnostic Criteria for Temporomandibular Disorders, J. Oral Facial Pain Headache, 29: 135-144.

Reiter, S., et al., 2018. Pain Catastrophizing and Pain Persistence in Temporomandibular Disorder Patients, J. Oral Facial Pain Headache, 32: 309-320.

Reiter, S., I. Eli, A. Gavish, and E. Winocur, 2006. Ethnic Differences in Temporomandibular Disorders Between Jewish and Arab Populations in Israel According to RDC/TMD Evaluation. J. Orofac Pain., 20: 36-42.

Schiffman, E., et al., 2014. Diagnostic criteria for temporomandibular disorders (DC/TMD) for clinical and research applications: recommendations of the international RDC/TMD consortium network and orofacial pain special interest group. J Oral Facial Pain Headache, 28: 6-27.

Schiffman, E., et al., 2014. Diagnostic criteria for temporomandibular disorders (DC/TMD) for clinical and research applications: recommendations of the international RDC/TMD consortium network and orofacial pain special interest group. J Oral Facial Pain Headache, 28: 6-27.

Segel-Karpas, D., L. Ayalonb, and M.E. Lachmanc, 2018. Retirement and depressive symptoms: A 10-year cross-lagged analysis. Psychiatry Res., 269: 565-570.

Song, Y.L., and A.U. Yap, 2018. Outcomes of therapeutic TMD interventions on oral health related quality of life: A qualitative systematic review. Quintessence Int., 49: 487-496. 
Steenks, M.H., and A. De Wijer, 2009. Validity of the Research Diagnostic Criteria for Temporomandibular Disorders Axis I in clinical and research settings. J. Oro. fac. Pain, 23: 927.

Valesan, L.F., et al., 2021. Prevalence of temporomandibular joint disorders: a systematic review and meta-analysis. Clin. Oral Investig, 25: 441-453.

Wang, M., 2007. Profiling retirees in the retirement transition and adjustment process: Examining the longitudinal change patterns of retirees' psychological well-being. J. Appl. Psychol., 92: 455474.

Wang, M., and K.S. Shultz, 2010. Employee retirement: A review and recommendations for future investigation. JOM. , 36: 172-206.

Winocur, E., M. Steinkeller-Dekel, S. Reiter, and I. Eli, 2009. A retrospective analysis of temporomandibular findings among Israeli-born patients based on the RDCTMD. J Oral Rehabil, 36: 11-17.

Yap, A.U., E.K. Chua, S.F. Dworkin, H. Tan, and K.B. Tan, 2002. Multiple pains and psychosocial functioning/psychologic distress inTMD patients; Int. J. Prosthodont, 15: 461-466.

Yekkalam, N., and A. Wänman, 2014. Prevalence of signs and symptoms indicative of temporomandibular disorders and headaches in 35-, 50-, 65- and 75-year-olds living in Västerbotten Sweden. Acta. Odontol. Scand., 72: 458-465.

Zaniboni, S., G. Topa, and C. Balducci, 2021. Core Self-Evaluations Affecting Retirement-Related Outcomes. Int. J. Environ. Res. Public Health, 18(174) : 1-15. 